\title{
Development of Faint Motion Recognition System Based on Improved Objects Tracking Method from Thermal Camera Images
}

\author{
Hanbyul Chae, Juhyun Lee, and Kicheon Hong
}

\begin{abstract}
This paper discusses an improved faint detection system. In the existing faint detection systems, tracking often fails if the object being tracked suddenly changes in its direction or shape. Authors propose a method to re-track the object for which tracking has failed in order to improve the accuracy and persistency of tracking performance. Therefore, it is possible to continuously track a certain senior citizen who lives alone by combining the tracking algorithm of Kalman filter and Camshift and detecting algorithm of blob labeling for images input from a thermal image camera. We propose a faint detection and response system based on the size change of the tracked trajectory and the change time. It can be found that the proposed system shows accurate faint detection through tracking.
\end{abstract}

Index Terms-Faint detection, object tracking, Kalman filter, thermal image.

\section{INTRODUCTION}

Person injury or emergency may occur due to impact when a senior citizen who lives alone or a patient becomes unconscious. To prevent and fast respond to an accident through faint detection, it is required to accurately track the object day and night and re-track the object even if tracking fails by using a thermal image camera.

Wai Kit Wong proposed faint monitoring by using a simple faint detection algorithm through a thermal image camera [1]. This theory, however, depends on a simple search algorithm and cannot show good performance if the field condition is complex.

M. R. Narayanan Lord proposed a detection method in which the user wears a sensor and presses a button to notify [2]. However, if the user forgets to wear the sensor or suddenly faints, not pressing the button, effective response is not possible.

V. Cantoni proposed a notification method by performing a certain action [3] but response is not possible in a faint situation. Thermal image can provide good results in identifying and tracking a moving person if used with an application program for detection when there is a significant difference in temperature between the background and the human. Another advantage of a thermal image camera is that it is more suitable than a color camera for motion tracking [4] as it can detect a bottleneck shadow or illuminating light in

Manuscript received January 4, 2015; revised June 12, 2015

Hanbyul Chae is with Information and Telecommunications Engineering, Suwon University, Korea.

Juhyun Lee is with Bit Computer Corporation, South Korea.

Kicheon Hong is with the University of Suwon, Republic of Korea (corresponding author; e-mail: kchong@suwon.ac.kr). most of the above motion tracking systems by using visible wave. As the output of a thermal image camera is a multilevel image which ensures smaller amount of data processing than that of a color camera, intelligent processing is required to accomplish good performance.

In this paper, an object is extracted by using the difference between two consecutive frames and the extracted object is continuously tracked by using Kalman Filter and Camshift algorithms. If the object is not tracked due to rapid change, it is re-tracked by getting the information on the detected object through Blob Labeling. We propose a response system where a faint situation is detected according to the analysis on the size change of the tracked course and change time, an alarm is triggered if a faint situation occurs and notification is made to the situation room or monitoring personnel.

This paper is constructed as follows. Section II describes Kalman Filter and Camshift tracking algorithms and detection algorithm by using Blob Labeling. Section III describes a system model, re-tracking algorithm by using the combination of tracking algorithm and detection algorithm, definition of the faint situation. Last, Section IV explains the experiment and conclusion.

\section{KALMAN FILTER AND ALGORITHMS FOR THERMAL IMAGE MOTION TRACKING AND BLOB LABELING ALGORITHM FOR OBJECT DETECTION}

\section{A. Kalman Filter Algorithm}

Kalman Filter is a well-known method for motion prediction [5]. It is a recursive algorithm to obtain optimum estimations with the state vector error minimized in a linear dynamic system which is interfered with white Gaussian noise. Kalman Filter is divided into three steps of prediction, measurement and correction, efficient body tracking by Kalman Filter requires setting of an appropriate tracking model. This paper sets the state vector by using the center coordinates $(x, y)$ of the change amount $(\Delta X, \Delta Y)$ of the detected body between the previous and current frames.

The state vector of Kalman Filter at time $t$ is defined as follows.

$$
x(t)=[x, y, \Delta x, \Delta y]^{T}
$$

Kalman Filter evolves with the system state vector, $x(t)$ and time.

$$
x(t+1)=\phi(t) x(t)+\omega(t)
$$

$w(t)$ is Gaussian noise with covariance $Q(t)$ and mean 0 
and defined by covariance $Q(t)$.

$$
Q(t)=\left[\begin{array}{llll}
0 & 0 & 0 & 0 \\
0 & 0 & 0 & 0 \\
0 & 0 & 1 & 0 \\
0 & 0 & 0 & 1
\end{array}\right]
$$

The measurement vector is given.

$$
z(t)=H(t) x(t)+v(t)
$$

$V(t)$ is a Gaussian noise factor with covariance $R(t)$ and mean 0 and defined by covariance $R(t)$.

$$
R(t)=\left[\begin{array}{ll}
1 & 0 \\
0 & 1
\end{array}\right]
$$

In this paper, the state change matrix $\varnothing(t)$ is defined on the assumption that the face moves in the straight direction at a constant speed.

$$
\varnothing(t)=\left[\begin{array}{llll}
1 & 0 & 1 & 0 \\
0 & 1 & 0 & 1 \\
0 & 0 & 1 & 0 \\
0 & 0 & 0 & 1
\end{array}\right]
$$

The input vector is a four-dimensional vector by using the center coordinates $(x, y)$ and defined by the measurement matrix $H(t)$ according to the change of $\Delta x$ and $\Delta y$ axes and the body.

$$
H(t)=\left[\begin{array}{cccc}
1 & 0 & \Delta t & 0 \\
0 & 1 & 0 & \Delta t \\
0 & 0 & 1 & 0 \\
0 & 0 & 0 & 1
\end{array}\right]
$$

\section{B. Camshift Algorithm}

Camshift algorithm is an improvement of Mean shift algorithm [6], a color segment method for use in a tracking environment and remedies the disadvantage of Mean shift by using the self-adjustment of the search window size. It is used to track an object at high speed, but has poor performance under lots of background noises and illuminance changes. By using the hue value distribution of the detected object area, change positions are predicted and detected and the center is searched to track the object.

The RGB color model is more sensitive to the illumination variance. Therefore, this algorithm converts the RGB color space into the hue of the HSV color model in order to reduce the impact of lighting on the tracked object.

Camshift algorithm is a transformation of the well-known meanshift algorithm and a search algorithm which operates in empirical distribution to extract a certain object.

Fig. 1 shows Camshift algorithm.

\section{Blob Labeling Algorithm}

Blob Labeling is a useful algorithm in detecting an object. In particular, thermal images have optimum conditions for performing Blob labeling as it can clearly separate an object from the background.

Blob Labeling algorithm requires binary coded images where the background and object are separated in a gray scale image. If neighboring pixel values are the same in a binary coded image, they are recognized and detected as one object. This paper covers 2-pass labeling. 2-pass labeling consists of the first pass for label arrangement and second pass for organizing arranged labels into correct sets.

Fig. 2 and Fig. 3 show 2-pass algorithm.

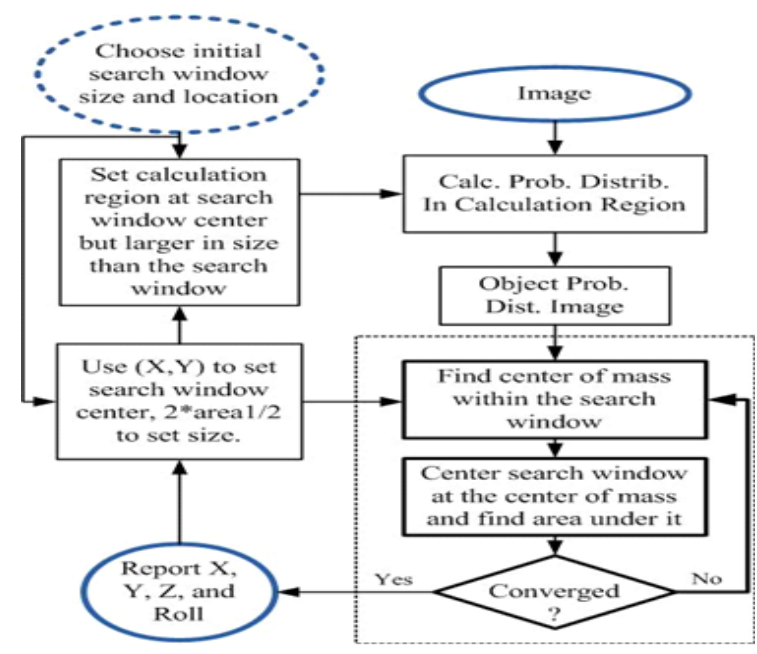

Fig. 1. Camshift algorithm flowchart.

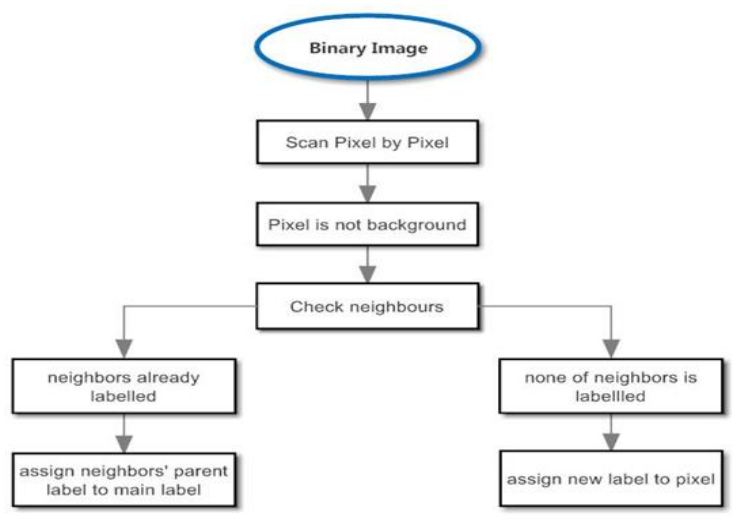

Fig. 2. First pass of blob labeling.

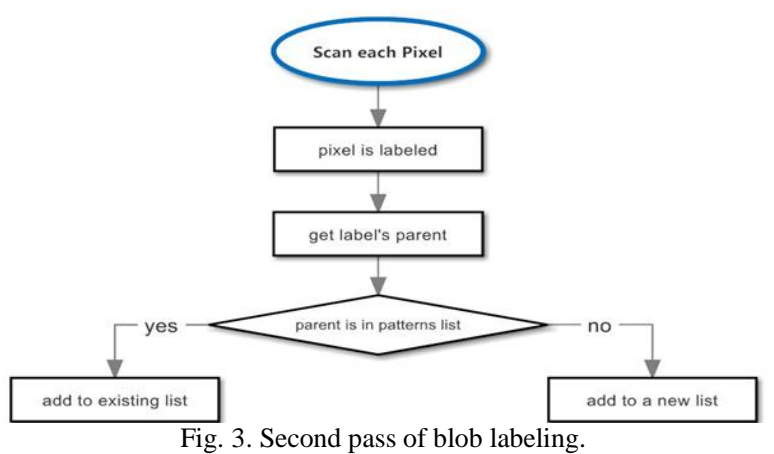

\section{FAint Detection System by Using the Size Change OF TRACKED COURSE AND CHANGE TIME}

\section{A. Structure of Faint Detection System}

Fig. 4 shows the faint detection system model which uses a thermal image camera proposed by this paper.

This system requires a thermal image camera which uses a precise resolution, laptop or PC and alarm signaling system. 


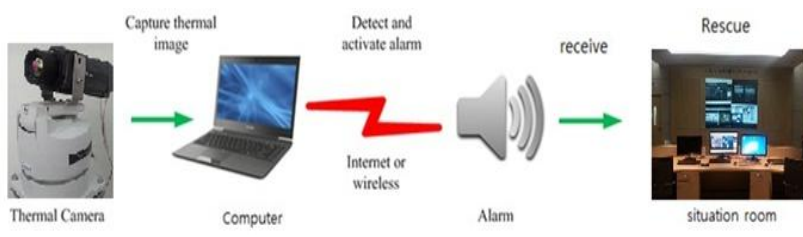

Fig. 4. Faint situation detection system model using a thermal camera.

\section{B. Characteristics of Thermal Camera}

Thermal Vision A300 manufactured by FLIR Systems thermal detection camera used in this paper - is a model which is capable of accurate temperature measurement. This camera has the scanning rate of $50 \mathrm{~Hz}$ and $60 \mathrm{~Hz}$, resolution of $320 \mathrm{X} 240$ pixels, and can detect temperature in the range of $+50 \mathrm{C}$ to $-15 \mathrm{C}$. It can capture an image by temperature. A300 can select a connection option. It can connect to the network through the router by using IEEE802.3 Ethernet connection, which makes it usable for making the best monitoring system.

\section{Object Detection}

The color and black-and-white images obtained by the thermal image camera are converted into 1-channel images. All other than pixel values within the range of a certain threshold are removed from the converted images and the resulting images are binary coded. Morphological technique is used to fill the hole. By performing Blob Labeling for these binary coded images, objects between the minimum and maximum sizes which a human can have are recognized and detected as individual objects.

\section{Re-tracking by Using Histogram Comparison}

If tracking has failed due to the loss of the object being tracked, histogram comparison is used to perform re-tracking.

Histogram information of the tracked object right before tracking has failed and that of detected objects for which separate detection is performed are compared to re-track the most similar object. For histogram comparison, Bhattacharyya distance calculation is used.

$$
d_{\text {Bhattacharyya }}\left(H_{1}, H_{2}\right)=\sqrt{1-\sum \frac{\sqrt{H_{1}(i), H_{2}(i)}}{\sqrt{\sum_{i} H_{1}(i) \sum_{i} H_{2}(i)}}}
$$

If histogram $H_{1}$ is completely identical to histogram $H_{2}, 0$ is returned. If they are only half-identical, 0.55 is returned. If they are completely different, 1 is returned.

Results of Tracked Object and Detecte Objects are as shown in Fig. 5.

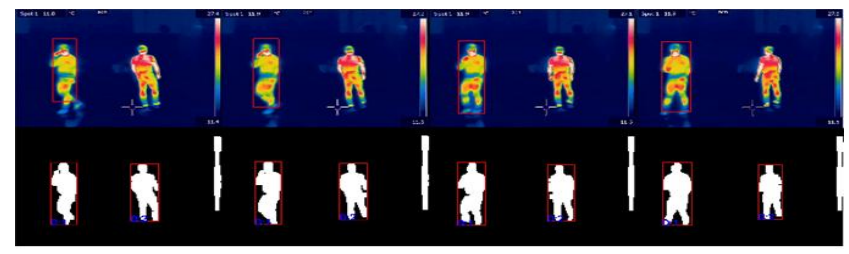

Fig. 5. Results of tracked object and detected objects.

$H_{t}=$ histogram of tracked object, $H_{1}, H_{2}=$ histogram of detected object

From the Bhattacharyya Distance results of $H_{1}$ and $H_{t}$, it can be found that two histograms are identical with the mean of about 0.055 . It can be found that $H_{2}$ and $H_{t}$ are about half identical as the mean of Bhattacharyya Distance results is 0.451 .

TABLE I: BHATTACHARYYA DisTANCE OF $\left(H_{t}, H_{1}\right)$ AND $\left(H_{t}, H_{2}\right)$

\begin{tabular}{lllll}
\hline \hline & $\begin{array}{l}\text { Frame } \\
109\end{array}$ & $\begin{array}{l}\text { Frame } \\
111\end{array}$ & $\begin{array}{l}\text { Frame } \\
113\end{array}$ & $\begin{array}{l}\text { Frame } \\
115\end{array}$ \\
\hline$d_{\text {Bhattacharyya }}\left(H_{t}, H_{1}\right)$ & 0.0908 & 0.0347 & 0.0466 & 0.0495 \\
$d_{\text {Bhattacharyya }}\left(H_{t}, H_{2}\right)$ & 0.4602 & 0.4473 & 0.4485 & 0.4518 \\
\hline \hline
\end{tabular}

\section{E. Flow of Faint Detection Algorithm}

Kalman Filter can appropriately track the object as it can successfully predict the state of the object in the next frame. Camshift algorithm can recognize a faint situation according to the change of the window size and the change time as it can adjust the window size according to the motion of objects [7]. The overall flow of faint recognition is as follows.

Step 1: Global parameter definition : global parameters used for the algorithm are defined. Hist $T_{T}=$ histogram of tracked object Hist $_{D}=$ histogram of detected object $H p=$ height of a person in the previous image, $H c=$ height of a person in the current image, $W p=$ width of a person in the previous image, $W c=$ width of a person in the current image, $f m=$ Frame number in which a person starts to bend, $f n=$ Frame number in which a person is laid down, $f=$ Accumulation of images where a person lies , $F=$ total frame (time) for determining if a person has fainted, $S=$ sectional frame (time) for determining if a person has fainted.

Step 2: Images are obtained by the thermal image camera and saved as RGB or Gray images. Objects are extracted from the difference between the current and previous frames and marked with rectangular frames.

Step 3: Kalman Filter performs prediction. Kalman Filter prediction is used to determine the initial position of the search window of Camshift algorithm and Camshift performs tracking. At this time, histogram information of the tracked objects is saved in real time to Hist ${ }_{t}$.

Step 4: Separate object detection is performed at the same time as tracking. Images obtained in step 2 are converted into gray area and binary coded. Gray and binary images are expressed as $G(x, y)$ and $g(x, y)$, respectively. Threshold indicates minimum temperature which a human can have.

$$
g(x, y)=\left\{\begin{array}{l}
0, G(x, y) \leq \text { Threshold } \\
1, \text { otherwise }
\end{array}\right.
$$

Step 5: Morphological technique is applied to binary coded images to fill holes and remove noise.

Step 6: Objects between minimum and maximum sizes which a human can have are detected by performing Blob Labeling for binary coded images. Histograms of detected objects are saved in $\left.\operatorname{Hist}_{D}[i]\right|_{i=0} ^{i=k-1}(k=$ number of detected objects).

Step 7: If an object is lost in step 3, Hist ${ }_{T}$ and Hist $\left._{D}[i]\right|_{\substack{i=k-1 \\ i=0}} ^{i}$ are compared and the most similar object is tracked again.

Step 8: The size of the tracking window is calculated and faint detection is performed. 
If $H c<W c, W c=W p, H c=H p, f>=F$ and $f n-f m<S$, the person is considered as having fainted.

Step 9: If a faint is detected, an alarm is triggered to start emergency rescue and the system is manually reset.

\section{EXPERIMENT AND CONCLUSION}

\section{A. Results and Comparison about Re-tracking} Performance

A faint situation can only be detected accurately when the object of interest can be re-tracked even if there are multiple objects in a room. This paper has enabled re-tracking of the object of interest by applying a re-tracking algorithm. Re-tracking performance results are as shown in Fig. 6 and Fig. 7.

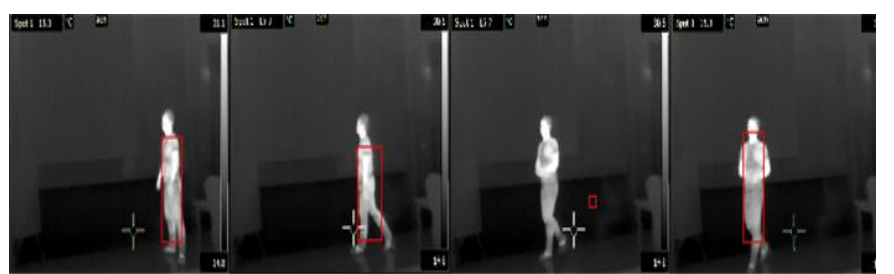

Fig. 6. Result of re-tracking in single object situation.

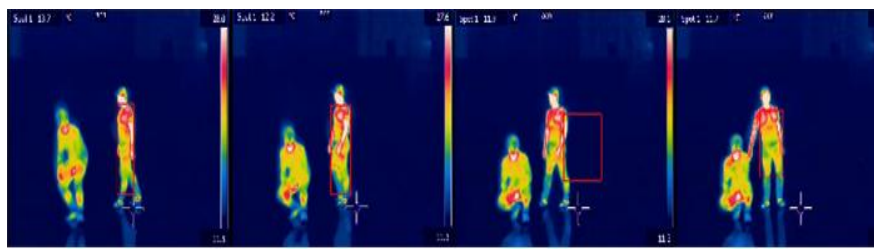

Fig. 7. Result of re-tracking in multiple object situation.

TABLE II: SUCCESS RATE OF RE-TRACKING OBJECT

\begin{tabular}{lcc}
\hline \hline & Number of images & Success rate \\
\hline Single Object Situation & 0.0908 & 0.0347 \\
Multiple Object Situation & 0.4602 & 0.4473 \\
\hline \hline
\end{tabular}

While re-tracking success rate is high due to excellent detection capability in single-object images, detection capability in multiple-object images is decreased as there are many difficult situations such as overlapped objects.

In proportion to this, re-tracking success rate is decreased.

Performances of the previously and currently proposed algorithms have been compared. Comparison results are shown in Fig. 8 and Fig. 9.

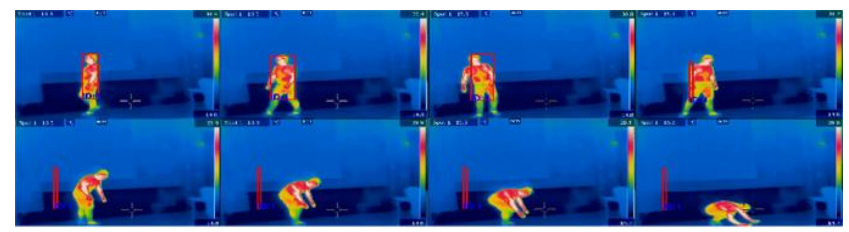

Fig. 8. Performance of the existing algorithms.

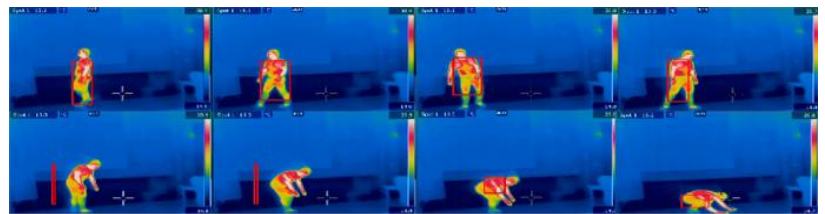

Fig. 9. Performance of the proposed algorithms.

In the previously proposed algorithm, an object being tracked is often lost if rapid change in shape or direction has occurred. This paper has remedied this weakness and improved tracking performance by ensuring that an object being tracked can be re-tracked even if it is lost."

\section{B. Results of Experiment on Difference between Faint and Lying Situations}

Results of experiments on difference between faint and lying situations are shown in frames. Images used for this paper have been shot for women in their twenties. For spontaneous lying and faint situations, images made by cutting in frames are shown in Fig. 10 and Fig. 11, respectively. Results about alarm notification after faint recognition are shown Fig. 12.

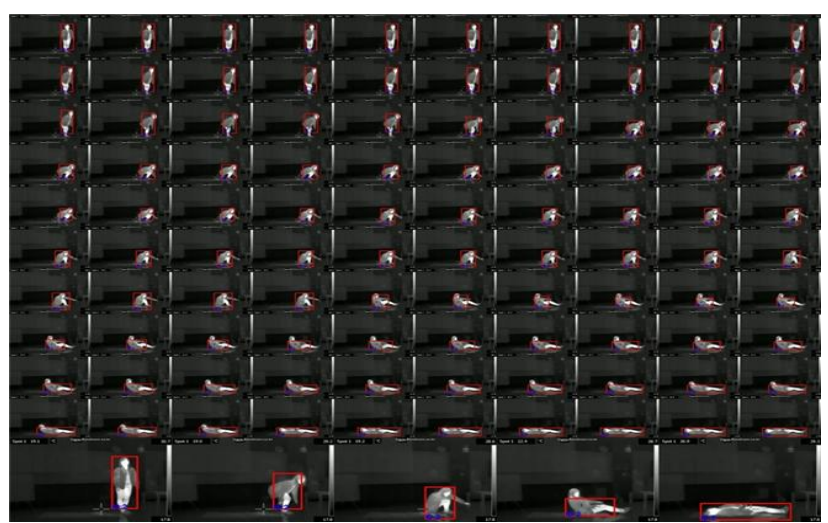

Fig. 10. Frame changes in the willingly lying down situation.

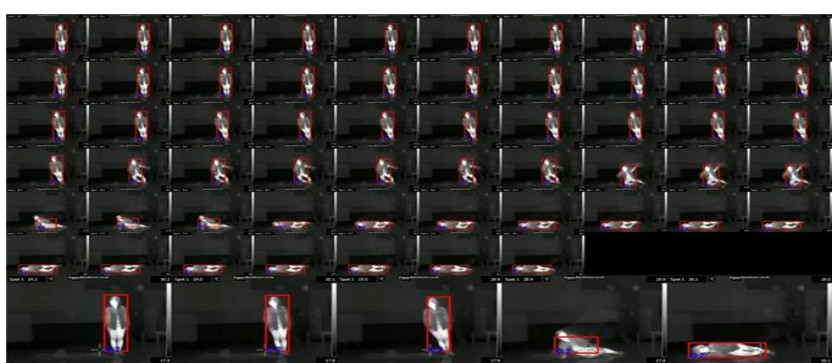

Fig. 11. Frame changes in the faint situation.

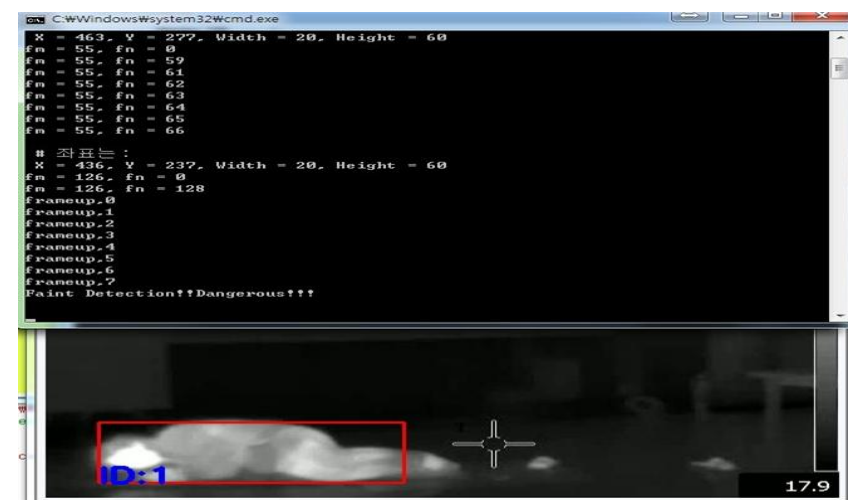

Fig. 12. Alarm on faint situation detection

The frame per second of these images is 29.7. 100 frames (2.97 seconds) were taken for normal lying and relatively short 57 frames (1.88 seconds) were taken for faint situations due to the absence of bending and crouching procedure.

As this paper covers faint detection targeted for senior citizens, it has been judged that senior citizens cannot lie faster than women in 20's and images have been shot for normal speed lying. Although images have been shot for many people, spontaneous lying has not exceeded 85 100 frames. Based on this result, it has been assumed that senior citizens would normally exceed 90 frames. It has been expected that both men in 20's and senior citizens show 
similar period of time for getting unconscious, not keeping posture and falling in a faint situation, and the system has been set to ensure that below 60 frames (about 2 seconds), a value higher than the test result is detected as a faint situation.

TABLE III: COMPARISON OF AVERAGE NUMBER OF FRAMES BETWEEN NORMAL LYING AND FAINT SiTUATIONS AND DETECTION SUCCESS RATES

\begin{tabular}{lllll}
\hline \hline & $\begin{array}{l}\text { Number of } \\
\text { frames for } \\
\text { normal lying }\end{array}$ & $\begin{array}{l}\text { Number of } \\
\text { frames for } \\
\text { faint situations }\end{array}$ & $\begin{array}{l}\text { Number } \\
\text { of images }\end{array}$ & $\begin{array}{l}\text { Faint } \\
\text { detection } \\
\text { success rate }\end{array}$ \\
\hline Men & 83.3 & 41.5 & 500 & $86 \%$ \\
Women & 92.6 & 46 & 500 & $81 \%$ \\
\hline \hline
\end{tabular}

\section{Conclusion}

This paper has proposed a stronger algorithm by adding a re-tracking algorithm to the strong faint detection in the previous proposal. As object tracking results are to be used to analyze and detect a faint situation, objects are extracted from the differences between two consecutive frames of the previous frames and the next frames and tracked by using Kalman Filter and Camshift algorithms. And, re-tracking capability has been added in combination with the detection algorithm by using Blob Labeling to improve persistency and accuracy.

Tracking performance was also compared to ensure correct faint detection. Based on these improvements, this paper has proposed a system for tracking senior citizens who live alone by combining the tracking algorithm which uses Kalman Filter and Camshift for images input from the thermal image camera and the detection algorithm which uses Blob Labeling, recognizing faint situations according to the change of the size of the tracked course and the change time and responding to them. The proposed system has shown continuous tracking and accurate faint detection capability.

Re-tracking capability of the Blob Labeling algorithm is decreased, however, due to significantly decreased detection capability if two or more objects are overlapped. It has been also found that the system, assumed as constructed with one camera, cannot ensure accurate faint detection if an object falls forward or backward, looking at the camera.

As a solution to this problem, it is expected that detection failure can be decreased by applying a more accurate detection algorithm and using a stereo camera system to remove blind spots through simultaneous monitoring from various directions. It is expected that falling at the front of the camera can be detected by a camera in another direction and the system can be triggered for fast response. Future study will discuss the development of an algorithm and construction of a system which can ensure high detection rate by using just one camera, instead of a stereo camera system.

\section{ACKNOWLEDGMENT}

This work was supported by the GRRC program of
Gyeonggi Province, Korea [(GRRC SUWON 2014-B3), Development of cloud Computing-based Intelligent Video Security Surveillance System with Active Tracking Technology]. Their support is gratefully acknowledged..

\section{REFERENCES}

[1] W. K. Wong, H. L. Lim, C. K. Loo, and W. S. Lim, "Home alone faint detection surveillance system using thermal camera," in Proc. 2010 Second International Conference on Computer Research and Development, May 2010, pp. 747-751.

[2] N. M. R. Lord et al., "Falls management: Detection and prevention, using a waist-mounted triaxial accelerometer," in Proc. $29^{\text {th }}$ Annual International Conference of the IEEE Engineering in Medicine and Biology Society, 2007, pp. 4037-4040.

[3] V. Cantoni, R. Marmo, and M. Zemblini, "Video surveillance and SOS request," in Proc. 14th International Conference on Image Analysis and Processing, 2007, pp. 560-565.

[4] C. N. Padole and L. A. Alexandre, "Motion based particle filter for human tracking with thermal imaging," in Proc. 3rd International Conference on Emerging Trends in Engineering and Technology (ICETET), Sept. 2010, pp. 158-162.

[5] R. E. A Kalman, "New approach to linear filtering and prediction problems," Transaction of the ASME - Journal of Basic Engineering, pp. 35-45, 1960.

[6] G. Bradski, "Real time face and object tracking as a component of a perceptual user intezrface," in Proc. 4th IEEE Workshop Applications of Computer Vision, 1998, pp. 214-219.

[7] J. Lee, X. Jiang, and K. Hong, "A robust faint situation recognition and response system based on object tracking algorithms using thermal camera," Computer Science and Its Applications Lecture Notes in Electrical Engineering, vol. 330, pp. 1233-1243, 2015.

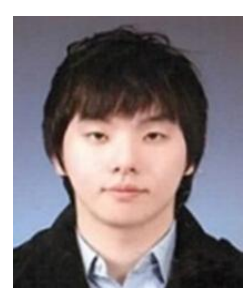

Hanbyul Chae received the BA degree in information and telecommunications engineering from Suwon University, Korea, in 2014. Hanbyul is currently a MA student at Information and Telecommunications Engineering, Suwon University, Korea. Mr. Chae research interests include image processing, signal processing, object tracking.

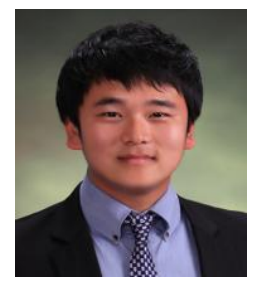

Juhyun Lee received the BA and MA degrees in information and telecommunications engineering from Suwon University, Korea, in 2013 and 2015. Juhyun is currently working for Bit Computer Corporation, South Korea. Mr. Lee research interests include image processing, signal processing, embedded system.

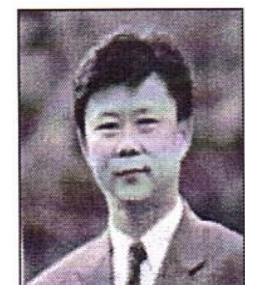

Kicheon Hong received the BA in electronics engineering from Sungkyunkwan University, Seoul, Korea in 1985 and the MA and Ph.D degrees in electrical engineering from Stevens Institute of Technology in 1988 and 1994, respectively. He was with Bell Communications Research Center (Bellcore) from 1992 to 1993 and Samsung Semiconductor Co. Ltd. From 1994 to 1998.

Kicheon has been with the Department of Information and Telecommunications Engineering, the University of Suwon, Seoul, Korea, where he is currently a professor. Prof. Hong research interests include image processing and compression, multimedia processor, intelligent surveillance system, embedded system. 